\title{
GESTÃO ESTRATÉGICA EMPRESARIAL: PROPOSIÇÃO DE UM MODELO DE MONITORAMENTO INFORMACIONAL NA ERA DO BIG DATA
}

\author{
BUSINESS STRATEGIC MANAGEMENT: \\ PROPOSAL OF A MODEL OF INFORMATIONAL MONITORING IN THE ERA OF BIG DATA \\ GESTIÓN ESTRATEGICA EMPRESARIAL: \\ PROPUESTA DE UM MODELO DE MONITOREO INFORMATIVO EM LA ERA DE BIG DATA
}

\footnotetext{
${ }^{1}$ Orandi Mina Falsarella, ${ }^{1}$ Celeste Aída Sirotheau Corrêa Jannuzzi, ${ }^{1}$ Cibele Roberta Sugahara

${ }^{1}$ Pontifícia Universidade Católica de Campinas
}

\section{Correspondência}

1 Orandi Mina Falsarella

Pontifícia Universidade Católica de Campinas

Campinas, SP

E-mail: orandi@puc-campinas.edu.br

ORCID:

Submetido em: 03-10-2016

Aceito em: 20-03-2017

Publicado: 04-04-2017

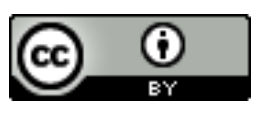

JITA: LP. Intelligent agents. 
RESUMO: Este trabalho tem como objetivo propor um Modelo de Monitoramento Informacional que possa subsidiar a gestão estratégica empresarial, de modo a identificar ameaças e oportunidades e forças e fraquezas, propiciando a obtenção de informações importantes para a elaboração e acompanhamento do Planejamento Estratégico Empresarial. Sobre o aspecto metodológico, trata-se de uma discussão de natureza teórica, fundamenta na pesquisa bibliográfica que procura recuperar conceitos relacionados a Big Data e Agente de Software Inteligente, identificando características que, se automatizadas, podem contribuir com o processo de busca e análise de dados e informações relevantes para a elaboração e acompanhamento do Planejamento Estratégico Empresarial. Por fim, demonstra-se que na interseção desses conceitos é possível observar que um Sistema de Inteligência Organizacional e Competitiva pode ser ampliado e evoluído ao utilizar as características de Big Data e Agente de Software Inteligente, resultando no Modelo proposto.

PalavraS-Chave: Gestão Estratégica. Inteligência Organizacional e Competitiva. Agente de Software Inteligente. Big Data.

ABSTRACT: This paper aims to propose an Informational Monitoring Model that can support the business strategic management, in order to identify threats and opportunities, and strengths and weaknesses, providing the obtainment of important information for the preparation and monitoring of the Business Strategic Planning. Under the methodological aspect, it is a theoretical discussion based on literature that seeks to recover concepts related to Big Data and Intelligent Software Agent, identifying characteristics that if automated, can contribute to the process of search and analysis of relevant data and information for the preparation and monitoring of the Business Strategic Planning. Finally, it demonstrates that in the intersection of these concepts is possible to observe a system of Competitive Intelligence and Organizational that can be expanded and evolved to use the features of Big Data and Intelligent Software Agent, allowing one to build as a result of the discussion, the model proposed.

KEYWORDS: Strategic Management. Organizational and Competitive Intelligence. Intelligent Software Agent. Big Data.

RESUMEN: Este estudio tiene el objetivo de proponer un modelo de Monitoreo Informativo que pueden apoyar la gestión estratégica de negocios, con la finalidad de identificar las amenazas y oportunidades, y las fortalezas y debilidades, proporcionando la obtención de información importante para la preparación y monitoreo de el plan estratégico corporativo. A la luz de los enfoques teóricos que se hace referencia en este estudio fue posible recuperar conceptos relacionados con Big Data y el Agente de Software Inteligente, la identificación de las características que si automatizado, puede contribuir al proceso de búsqueda y análisis de datos e información relevantes para la preparación y monitoreo de un plan estratégico de negocio. Los resultados señalan que con la intersección de estos conceptos un Sistema de Inteligencia Competitiva y Organizacional se puede ampliar mediante el uso de las características de las funciones de Big Data y el Agente de Software Inteligente, que le permite construir el modelo propuesto.

PALABRAS CLAVE: Gestión Estratégica. Inteligencia Organizacional y Competitiva. Agente de Intelligent Software. Big Data.

\begin{tabular}{c|c|c|c|c|c}
\hline (C) RDBCI: Rev. Digit. Bibliotecon. Cienc. Inf. & Campinas, SP & v.15 & n.2 & p. 420-441 & maio/ago. 2017 \\
\hline
\end{tabular}




\section{INTRODUÇÃ̃o}

Planejamento Estratégico Empresarial (PEE) e Tecnologia da Informação e Comunicação (TIC) são assuntos muito abordados na literatura técnica e científica há vários anos. Muitos trabalhos que exploram esses conceitos foram e ainda serão apresentados com o propósito de as TIC agregarem valor às estratégias de negócio, procurando trazer para as organizações vantagens ou diferenciais competitivos.

Quando se pensa no PEE sabe-se que ele está relacionado diretamente à gestão estratégica de uma organização, pois é a partir dele que se tem a definição da Visão e da Missão da Instituição, a fixação dos Objetivos e Metas e das Estratégias a serem utilizadas para esse fim, bem como a indicação dos Recursos necessários, sejam eles Financeiros, Materiais ou Humanos, tudo para que a organização se torne competitiva (THOMPSON et al., 2008).

Nesse contexto, buscando reforçar a efetividade da gestão estratégica, os estudos sobre a inteligência nas organizações ganharam profundidade contribuindo para o surgimento de conceitos como Inteligência Organizacional (IO) e Inteligência Competitiva (IC). O primeiro, Inteligência Organizacional, segundo Starec, Gomes e Bezerra (2005) referem-se ao gerenciamento estratégico da informação, proporcionando respostas para que as decisões possam ser tomadas quase que instantaneamente. O segundo conceito, ou seja, o de Inteligência Competitiva, Oliveira (2004) define como aquela que monitora o ambiente externo na busca de ameaças e oportunidades com o propósito de a organização alcançar vantagem competitiva perante os concorrentes.

Assim, sob esse prisma e pensando na elaboração do PEE, um questionamento que pode ser feito é se a Inteligência Organizacional e/ou a Inteligência Competitiva só se preocupam com informações externas ao analisar ameaças e oportunidades, não olhando para as informações internas importantes para a descoberta de forças e fraquezas. Valentim et al. (2003, p.8) respondem a esse questionamento quando afirmam, por exemplo, que a Inteligência Competitiva procura pesquisar "o ambiente onde a empresa está inserida, com o propósito de descobrir oportunidades e reduzir os riscos, bem como diagnostica o ambiente interno organizacional, visando o estabelecimento de estratégias de ação a curto, médio e longo prazo".

Segundo Falsarella et al. (2014), o reconhecimento da importância da Inteligência Organizacional e da Inteligência Competitiva é determinante na gestão das organizações. Os autores entendem que elas se complementam em suas proposições e favorecem as organizações na obtenção de vantagem competitiva, constituindo, então, um conceito mais amplo chamado de Inteligência Organizacional e Competitiva (IOC).

A IOC pode ser determinante para o Planejamento Estratégico Empresarial no monitoramento do ambiente interno e externo da organização, contribuindo, quando

\begin{tabular}{l|l|l|l|l|l}
\hline (C) RDBCI: Rev. Digit. Bibliotecon. Cienc. Inf. & Campinas, SP & v.15 & n.2 & p. 420-441 & maio/ago. 2017 \\
\hline
\end{tabular}


necessário, para a correção ou mudança de suas estratégias. Mas, para que isso aconteça, é essencial que as mudanças de contexto onde a instituição atua sejam rapidamente observadas. Mais do que isso, para Demirkan e Delen (2013), no mundo dos negócios, as organizações precisam ser capazes de responder rapidamente às necessidades de mudanças do mercado.

Assim, dados e informações estão se tornando bens primários para muitas organizações, fazendo com que a maioria delas busque coletá-los e processá-los o mais rapidamente possível, independente do desafio atualmente existente de gerenciar grandes volumes de dados. Segundo Begoli e Horey (2012), nos últimos anos, grandes quantidades de dados estão cada vez mais disponíveis em volumes significativos (petabytes ou mais). Elas são provenientes de muitas fontes como: redes sociais; mídias sociais; chamadas de aparelhos móveis; atividades científicas; simulações; experimentos; sensores ambientais; entre outros, além, obviamente, de fontes tradicionais.

Ji et al. (2012) exemplificam esse fato quando afirmam, em estudo realizado no início desta década, que o Facebook fornece 570 bilhões de páginas Web e armazena 3 bilhões de novas fotos a cada mês, além de administrar 25 bilhões de partes de conteúdos. Diante desse enorme e crescente volume de dados e informações é que se delineia um grande problema: como as organizações podem fazer uma observação constante e contínua de seu ambiente, de modo a diminuir o tempo de latência do processo decisório, face ao grande volume de dados e de informações produzido atualmente?

É nesse contexto, como resposta à essa situação, que surge na literatura científica e empresarial um conceito mais amplo que recebe a denominação de Big Data. Para Han et al. (2012), Big Data são os dados que devem ser analisados pelas empresas, mas que não foram objeto de análise por não terem sido possíveis processá-los por meio da tecnologia atualmente existente.

De acordo com os autores, no uso do Big Data, além da coleta e análise de um volume expressivo de dados/informações, é possível também trabalhar com uma grande variedade de formatos (imagens, sons, textos etc.). Os resultados das informações obtidas são apresentados de forma sequenciada, auxiliando a tomada de decisão, por exemplo, na organização.

Conforme Agarwal et al. (2013), para extrair insights de grandes volumes de dados, as informações precisam ser correlacionadas ou harmonizadas para um nível comum de granularidade. Os autores complementam que muitas informações valiosas somente são encontradas quando elas são provenientes de fontes externas e são integradas com as informações internas que residem principalmente em bancos de dados relacionais.

Assim, voltando à afirmação de Falsarella et al. (2014) de que a IOC pode contribuir com a elaboração e acompanhamento do Planejamento Estratégico Empresarial, percebe-se 
que dentro do contexto da gestão estratégica, os conceitos Big Data e IOC estão diretamente relacionados e são complementares entre si. Em conjunto, eles podem contribuir com o PEE ao buscar e fornecer dados e informações relevantes, além de insights ao monitorar e observar os ambientes interno e externo de uma organização, na busca de forças e fraquezas, como também de ameaças e oportunidades.

A partir dessa associação, e pelo fato de estar relacionado aos conceitos supracitados, destaca-se um outro conceito que também deve ser acrescido à essa discussão: o de Agente de Software Inteligente. Definido como uma entidade computacional, com características de mobilidade e inteligência, entre outras, o Agente de Software Inteligente tem a finalidade de navegar pela rede de computadores a pedido de seu usuário, buscando e coletando dados e informações, fazendo análises e identificando os que são relevantes.

Maes apud Duan et al. (2012) reforçam que

[...] o aparecimento do agente de software inteligente, como um conceito e uma tecnologia, é uma das soluções para reduzir os problemas de sobrecarga de informação enfrentados pelas organizações empresariais contemporâneas, por serem entidades que realizam um conjunto de atividades em nome de um usuário, ou outro programa, com um grau de independência e autonomia, empregando algum conhecimento ou representando os objetivos e desejos do usuário (MAES apud DUAN et al., 2012, p. 5535, tradução nossa).

Assim, diante da discussão feita até o momento e diante do grande volume de dados e informações que precisam ser constantemente monitorados é que se desenvolve este trabalho cujo objetivo é propor um Modelo de Monitoramento Informacional que possa subsidiar a gestão estratégica empresarial, de modo a identificar ameaças e oportunidades e forças e fraquezas, extraindo informações importantes para a elaboração e acompanhamento do PEE.

\section{PROCEDIMENTO METOdOLógico}

Para o desenvolvimento deste trabalho, de natureza teórica, foi utilizada a pesquisa bibliográfica, uma vez que esta contribui para um pensamento reflexivo que permite descobrir novos fatos e relações em qualquer área de conhecimento (LAKATOS e MARCONI, 2007). A existência de diversas obras nacionais e internacionais sobre os assuntos abordados no trabalho, ou seja, Planejamento Estratégico Empresarial (PEE), Inteligência Organizacional, Inteligência Competitiva, Big Data e Agente de Software Inteligente, contribuíram para essa escolha, cujo principal parâmetro foi o período da publicação.

Os conceitos de PEE e IOC abordados nesta discussão foram recuperados de uma pesquisa bibliográfica realizada anteriormente, cujo trabalho já publicado fundamenta a continuidade da presente pesquisa. Esses conceitos foram utilizados neste trabalho na

\begin{tabular}{l|c|c|c|c|c}
\hline (C) RDBCI: Rev. Digit. Bibliotecon. Cienc. Inf. & Campinas, SP & v.15 & n.2 & p. 420-441 & maio/ago. 2017 \\
\hline
\end{tabular}


descoberta de possíveis relações com os conceitos de Big Data e Agente de Software Inteligente.

Os conceitos de PEE e IOC abordados nesta discussão foram recuperados de uma pesquisa bibliográfica realizada anteriormente, cujo trabalho - publicado em 2014 com o título Planejamento Estratégico Empresarial: proposta de um Sistema de Inteligência Organizacional e Competitiva (FALSARELLA; JANNUZZI; SUGAHARA, 2014) fundamenta a continuidade da presente pesquisa. Esses conceitos foram utilizados neste trabalho na descoberta de possíveis relações com os conceitos de Big Data e Agente de Software Inteligente.

Para o Agente de Software Inteligente (ASI) foram selecionadas especificamente as publicações sobre o assunto compreendendo os anos de 1999 a 2005, época que esse conceito surgiu com maior frequência na literatura. Quanto ao conceito de Big Data, por ser um assunto atual, o período da pesquisa compreendeu as publicações recentes, ou seja, a partir de 2011 quando as primeiras referências sobre o assunto surgiram.

No presente trabalho, apresenta-se inicialmente uma discussão sobre Big Data e Agente de Software Inteligente, descrevendo os principais conceitos e características. Posteriormente, buscando atingir o objetivo do trabalho, ou seja, propor o Modelo de Monitoramento Informacional, são detalhadas as relações desses conceitos e características com a Inteligência Organizacional e Competitiva e elaboração e acompanhamento do Planejamento Estratégico Empresarial.

\section{BIG DATA}

Impulsionado pela crescente popularidade das redes sociais, e-commerce e um aumento crescente na realização de negócios colaborativos, está ocorrendo uma explosão de dados. É nesse contexto que surge o Big Data como um conceito importante na gestão estratégica das organizações. Para Begoli e Horey (2012), Big Data refere-se à

[...] prática de coleta e processamento de grandes conjuntos de dados associados a sistemas e algoritmos usados para analisar esses conjuntos de dados massivos. Juntamente com a descoberta de conhecimento; a movimentação de grande volume de dados oferece muitas oportunidades únicas para as organizações se beneficiarem (em relação a novas ideias de negócios, otimizações etc.) (BEGOLI; HOREY, 2012, p. 215, tradução nossa).

Borkar et al. (2012) dizem que empresas de todas os tipos e tamanhos estão monitorando compras, pesquisas de produtos e outras informações para aumentar a eficácia do seu marketing. Governos e empresas estão rastreando o conteúdo de blogs e tweets para realizar análises de sentimentos. Organizações de saúde pública estão monitorando tweets e web para

\begin{tabular}{l|l|l|l|l|l}
\hline (C) RDBCI: Rev. Digit. Bibliotecon. Cienc. Inf. & Campinas, SP & v.15 & n.2 & p. 420-441 & maio/ago. 2017 \\
\hline
\end{tabular}


acompanhar o progresso e tendências de epidemias. Os cientistas sociais estão estudando tweets e redes sociais para entender como as informações se espalham e como podem ser mais eficazmente utilizadas. É neste contexto que poderosas ferramentas de análise podem ajudar as empresas a lidar com decisões complexas, oferecendo novos insights, criando um ciclo virtuoso (MITHAS et al., 2013).

Em seus estudos sobre o Big Data, Kaisleret al. (2013) afirmam que este é um fenômeno emergente caracterizado por três "Vs": Volume, Velocidade e Variedade. O Volume diz respeito ao aumento da quantidade de dados existente que de terabytes passou para pentabytes e está chegando a exabytes. Alguns especialistas estão sugerindo que zettabytes $\left(10^{21}\right)$ serão alcançados nos próximos anos. A Velocidade se preocupa não só com a rapidez com que os dados são acumulados, mas também com a rapidez que eles mudam. Já a Variedade se restringe a dados estruturados ou não estruturados.

Beulke (2011) considera mais dois "Vs", ou seja, Veracidade e Valor. Com relação à Veracidade o autor descreve que, como o volume de dados é muito grande, é certo que haverá entre eles alguns não confiáveis, que podem distorcer qualquer raciocínio que se pretenda fazer. Com relação a Valor sugere a capacidade de extrair dados que agreguem valor e façam sentido para a organização empresarial.

Singh e Singh (2012) complementam a característica de Valor quando afirmam que os dados devem ser utilizados de modo que o seu valor seja maximizado para os negócios, sendo, nesse caso, o tempo de coleta e análise muito crítico. Os autores afirmam ainda:

\footnotetext{
Quando uma empresa aproveita todas as informações disponíveis nos grandes volumes de dados em vez de apenas um de seus subconjuntos, ela passa a ter uma grande vantagem sobre os concorrentes no mercado. Nesse caso, Big Data pode ajudar a obter insights, permitindo que a instituição possa tomar as melhores decisões. Big Data apresenta uma oportunidade sem precedentes para criar vantagem competitiva e uma melhor prestação de serviços. Também exige nova infra estrutura e uma nova maneira de pensar sobre o modo como os negócios e as TIC serão conduzidos. O conceito de Big Data vai mudar a forma de como fazemos as coisas hoje (SINGH e SINGH, 2012, p. 1, tradução nossa).
}

A Figura 1 sintetiza os 5 Vs, relacionando: Volume à quantidade de dados existentes; Velocidade ao aumento e modificação constante dos dados; Variedade, aos dados estruturados e não estruturadas; Veracidade, ao extrair dados confiáveis, descartando os não confiáveis; e Valor à geração de insights quando da análise dos dados coletados.

\begin{tabular}{l|c|c|c|c|c}
\hline (C) RDBCI: Rev. Digit. Bibliotecon. Cienc. Inf. & Campinas, SP & v.15 & n.2 & p. 420-441 & maio/ago. 2017 \\
\hline
\end{tabular}




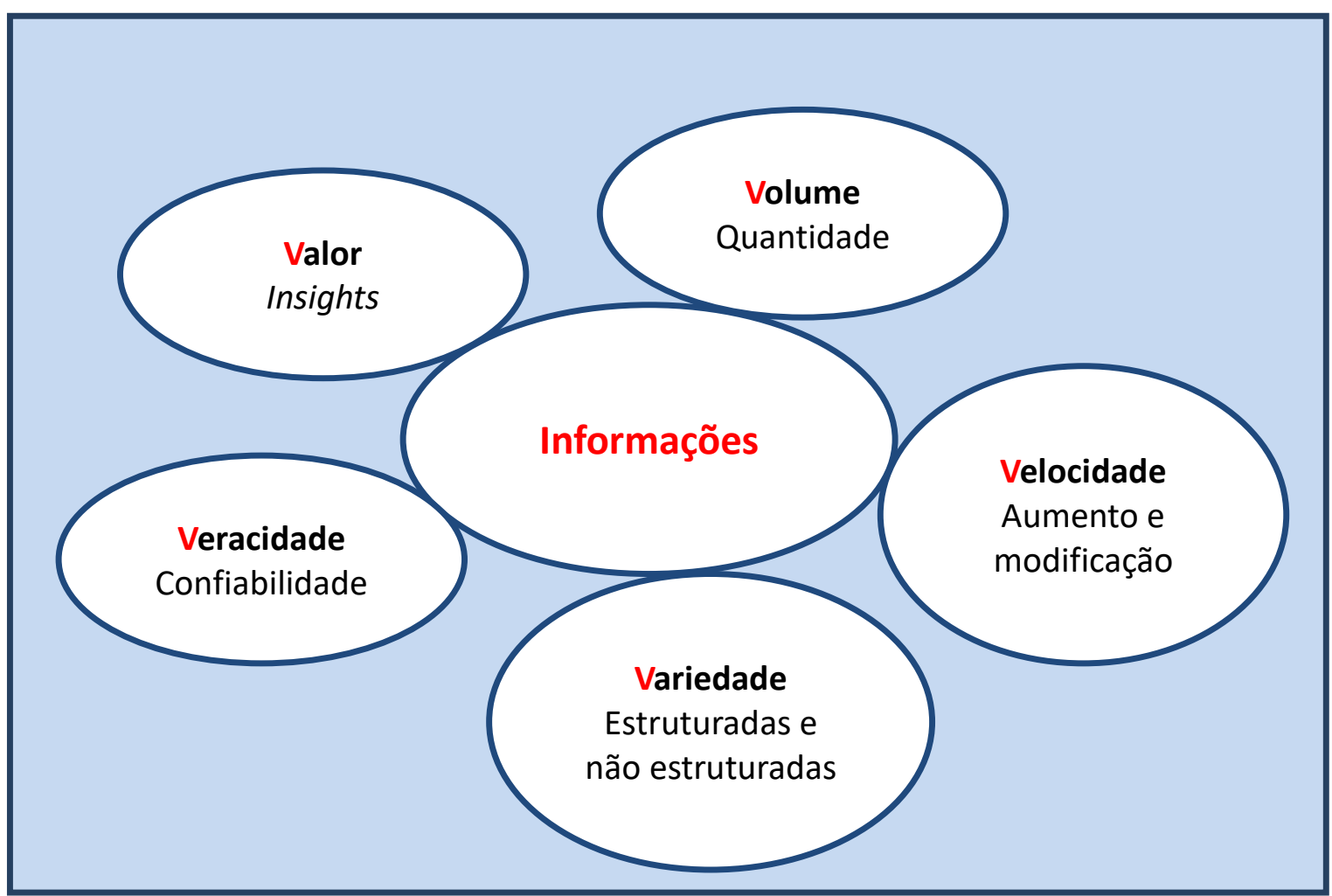

Figura 1 - Big Data 5 Vs

Fonte: Autores deste trabalho.

O conjunto de características expressas pelos 5 V's é que imprime ao Big Data sua importância conceitual no tratamento de dados. No entanto, por se tratar de um conceito há a necessidade da existência de ferramentas capazes de coletar os dados, analisá-los e disponibilizá-los, no sentido de subsidiar a gestão estratégica empresarial. Uma dessas ferramentas pode ser o agente de software inteligente que será apresentado a seguir.

\section{AGENTES DE SOFTWARE INTELIGENTES}

Um agente de software é um programa de computador que opera dentro de ambientes de computação. Os proprietários de agentes de software (usuários) podem instruir seus agentes para percorrer a rede, acessar informações desejadas através da troca de dados com outros agentes ou pessoas, e lidar com transações comerciais e pessoais (BARFIELD, 2006). Segundo Hickman (2010), um agente é uma entidade de software capaz de realizar pesquisas em unidades de armazenamento acessadas por meio de redes locais ou remotas.

Resende (2003) apresenta uma interessante taxonomia para os agentes que são representadas em quatro eixos. São eles: 
$\checkmark$ Cognitivo - um agente pode conter um modelo de representação interna do ambiente, um modelo racional de decisão ou agir a partir de um modelo de reações aos estímulos provocados pelo ambiente;

$\checkmark$ Foco - um agente pode possuir similaridades comportamentais com humanos;

$\checkmark$ Atuação - um agente pode atuar isoladamente ou interagir socialmente com outros agentes;

$\checkmark$ Ambiental-um agente pode atuar no desktop ou em uma rede (internet ou intranet).

Para Aron et al. (2004), com as tecnologias baseadas na WEB, é possível que empresas de vendas on-line possam obter informações precisas sobre compradores e oferecer produtos e serviços que satisfaçam as preferências individuais de cada consumidor. Isso é possível por meio da utilização de agentes de software inteligentes, ou seja, entidades computacionais que podem avaliar e estimar as preferências do comprador, analisar históricos de compra, além de apresentar estratégias de marketing personalizadas levando em conta preço, recomendação de produtos, entre outros.

Kvassov (2000) corrobora com essa afirmação quando enfatiza que a Internet contém dados/informações potencialmente importantes para uma organização. Isso tudo combinado com ferramentas inteligentes de recuperação e gestão podem fornecer para um gestor insights sobre as tendências do negócio detectadas no ambiente externo da organização.

Ao expressar esse entendimento, o autor refere-se aos Agentes de Softwares Inteligentes (ASI) como programas computacionais que podem navegar em ambientes de computação heterogêneos (redes de computadores e internet), acompanhado ou não de outros agentes, com o propósito de alcançar um objetivo específico. Segundo Kvassov (2000), um agente pode possuir as seguintes capacidades:

$\checkmark$ Coletar autonomamente na internet dados de diferentes fontes;

$\checkmark$ Mudar parâmetros de pesquisa, a fim de atingir objetivos específicos;

$\checkmark$ Realizar buscas sofisticadas que podem conter tanto parâmetros qualitativos como quantitativos;

$\checkmark$ Escolher os dados com base em seu conteúdo;

$\checkmark$ Consultar fontes de dados selecionadas regularmente;

$\checkmark$ Rastrear e extrair mudanças ao longo do tempo.

Para Jennings (2000), existem vários pontos sobre a definição e construção de Agentes de Softwares Inteligentes que precisam ser respeitados. São eles:

$\checkmark$ O problema a ser resolvido tem que estar bem identificado e com limites bem definidos; 
$\checkmark \quad \mathrm{O}$ ambiente onde ele vai agir deve estar definido. Ele age sobre o ambiente por meio de informações recebidas de sensores, ou seja, em resposta a estímulos;

$\checkmark$ Ele deve ser projetado para atender a uma finalidade específica, com objetivos e metas para alcançar;

$\checkmark$ Ele deve ter autonomia, ou seja, ter controle tanto sobre o seu estado interno como sobre o seu comportamento;

$\checkmark$ Ele deve ser reativo à mudança do ambiente e responder em tempo hábil, sendo capaz de antecipar objetivos futuros.

Os agentes de software contribuem para reduzir o trabalho humano, uma vez que as tarefas são executadas mais rapidamente e com melhor precisão ao lidar com a sobrecarga de informações, pois podem filtrar automaticamente grandes quantidades de informações não estruturadas disponíveis na rede (MUNTEAN; TOMA, 2009). Assim, pensando em mobilidade, os agentes podem ser:

$\checkmark$ Estáticos - são executados no local onde estão instalados por meio de programação prévia ou estímulos do ambiente. Por exemplo, ao final do dia, em um horário prédeterminado o agente verifica o resultado das vendas e informa seu usuário dos pontos de venda que não atingiram a meta diária. (programação prévia). No caso de estímulos, um exemplo seria as informações de vendas que ocorreram acima da média serem detectadas e informadas pelo agente ao usuário em tempo real, à medida que novos pedidos forem sendo inseridos no sistema;

$\checkmark$ Móveis - São agentes que navegam pela rede a pedido de seus usuários com tarefas a serem cumpridas. Por exemplo, um agente que visita sites de concorrentes com o propósito de comparar preços de produtos.

Para Néri (2005), agentes de software podem ser considerados programas aos quais são delegadas tarefas e poder de decisão, tais como definir qual a melhor compra a ser feita, quais os produtos são interessantes para oferecer a um cliente etc. No entanto, o autor pondera que, como todo software, agentes são formalizações do conhecimento humano, codificados em linguagem de computador que, sem bons mecanismos de decisão, não serão bons tomadores de decisão.

Outro conceito apresentado por Néri (2005) é o sistema multi agente que contempla vários agentes cooperando entre si, de modo que o sistema como um todo alcance o objetivo previamente estipulado. De acordo com o autor, a cooperação exige comunicação entre essas entidades de software.

Anderson e Rönnbom (2009) apresentam uma série de características que envolvem os agentes de software. A partir do entendimento que agentes diferentes devem ter capacidade de 
aprendizagem diferenciadas, os autores classificam essas características em três níveis: compulsório, típico e adicional.

Para os autores, as características compulsórias referem-se à autonomia do agente para decidir se vai realizar uma tarefa ou não; as características típicas, por sua vez, referem-se à forma de comunicação e cooperação do agente; e por fim, as características adicionais representadas pela confiabilidade, robustez etc. Os detalhes dessas características são representados pela Figura 2 e descritas a seguir:

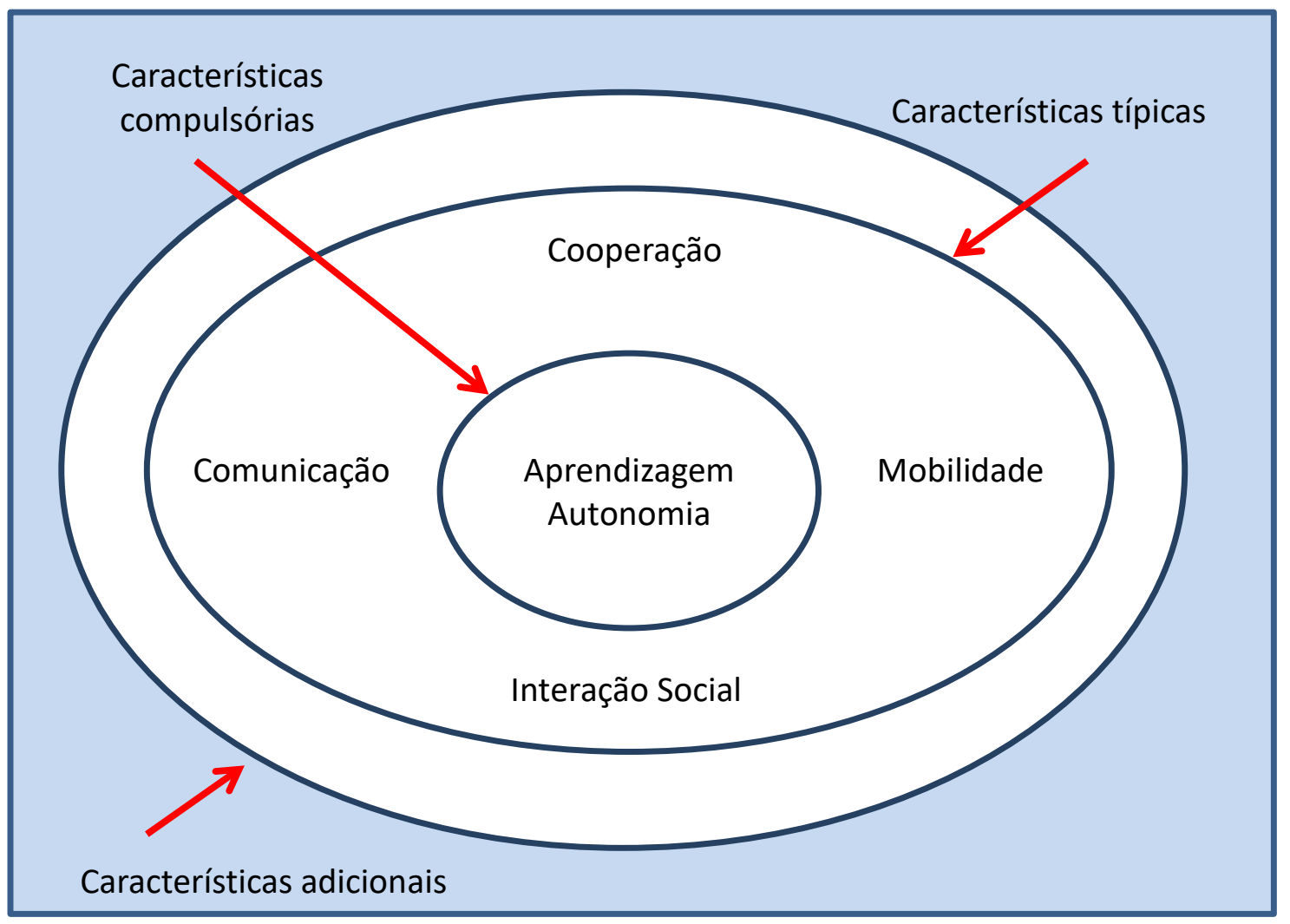

Figura 2 - Características dos Agentes de Software

Fonte: Adaptado de Anderson e Rönnbom, 2009.

Aprendizagem - $\mathrm{O}$ agente também deve ser capaz de aprender e usar um novo conhecimento para resolver novos problemas. $\mathrm{O}$ autor complementa afirmando que para ele ser capaz de aprender o agente está simulando uma importante característica humana que é ser inteligente. A aprendizagem é uma característica fundamental, pois o agente atua em um ambiente que é dinâmico e que muda constantemente;

$\checkmark$ Autonomia - o agente pode agir por conta própria e tomar suas próprias decisões, liberando o seu usuário para realizar outras tarefas mais interessantes. Segundo o autor, autonomia e inteligência são palavras consideradas sinônimas para outros autores. Ele define agente inteligente como uma entidade que tem um objetivo e foi criado para ajudar um usuário com uma tarefa específica em um domínio específico. 
Cooperação - o agente pode cooperar com outros agentes trocando informações. Desse modo, tarefas complexas podem ser subdivididas e realizadas por vários agentes, sendo, portanto, mais fáceis de serem realizadas;

$\checkmark$ Mobilidade - essa característica permite que o agente viaje por vários computadores existentes na rede e execute suas tarefas localmente em cada um deles. É como se um usuário atribuísse uma tarefa para um agente, desligasse seu computador e ao religá-lo no dia seguinte o agente retornasse à origem com a tarefa executada após ter visitado vários sites na WEB;

$\checkmark$ Comunicação - é a habilidade que o agente possui de se comunicar e trocar informações com outros agentes;

$\checkmark$ Interação Social - é o contexto de se criar a ilusão de que um agente é capaz de realizar interação social significativa com seres humanos. A criação em computador dessa característica requer uma grande variedade de tecnologias, incluindo a reconhecimento de voz, compreensão de linguagem natural, animações, entre outras.

Segundo Sproule e Archer (2000), dentro do campo dos Sistemas de Informação (SI), os agentes de software estão relacionados com o estudo dos Sistemas de Apoio à Decisão (SAD), como pode ser observado no Quadro 1.

Quadro 1 - Desenvolvimento de SAD e Agente de Software

\begin{tabular}{|c|c|}
\hline O desenvolvimento de um SAD deve: & O desenvolvimento de um agente de software deve: \\
\hline $\begin{array}{c}\text { Permitir a segmentação do processo de decisão } \\
\text { em componentes menores }\end{array}$ & $\begin{array}{c}\text { Possuir diferentes representações dividindo o problema e os } \\
\text { sistemas de raciocínio em partes menores }\end{array}$ \\
\hline Contemplar muitos níveis de interação com o & Permitir que o agente: \\
usuário & $\checkmark \quad$ aprenda com o usuário; \\
& $\checkmark \quad$ trate fatores e situações dinamicamente; \\
& $\checkmark \quad$ possua um comportamento de busca e escolha \\
\hline Ser um processo interativo com ferramentas & $\begin{array}{c}\text { Personalizar o agente para cada usuário, necessidade e } \\
\text { aplicação }\end{array}$ \\
\hline
\end{tabular}

Fonte: Adaptado de Sproule e Archer (2000).

A relação $\mathrm{SAD}$ com os agentes de software deve ser considerada, uma vez que os agentes podem ajudar os usuários fornecendo informações e subsídios para auxiliar no processo decisório. Complementando, a tomada de decisão envolve a busca de informações relevantes disponíveis no meio ambiente onde a instituição atua e a geração de soluções e alternativas que permitam ao usuário escolher e tomar a melhor decisão.

\begin{tabular}{l|c|c|c|c|c}
\hline (C) RDBCI: Rev. Digit. Bibliotecon. Cienc. Inf. & Campinas, SP & v.15 & n.2 & p. 420-441 & maio/ago. 2017 \\
\hline
\end{tabular}




\section{MODELO DE MONITORAMENTO INFORMACIONAL}

Retomando o objetivo deste trabalho que é propor um Modelo de Monitoramento Informacional, que possa subsidiar a gestão estratégica empresarial, de modo a identificar ameaças e oportunidades e forças e fraquezas, informações importantes para a elaboração e acompanhamento do PEE é preciso apresentar as etapas do Ciclo da Inteligência Organizacional e Competitiva descrito por Alves e Falsarella (2009) a seguir:

$\checkmark$ Planejamento - nessa etapa é determinado o campo de interesse que uma organização pretende observar, definida a demanda e as fontes de informação com o propósito de identificar que dados e informações são importantes e que podem fornecer subsídios à etapa de análise;

$\checkmark$ Coleta de dados e informações - tem início após a identificação das fontes de informação. Nessa etapa as bases de dados, sejam elas internas ou externas, são acessadas com o propósito de buscar informações relevantes e confiáveis;

$\checkmark$ Análise - é na análise dos dados e informações por meio da busca de relações que é gerada alguma inteligência;

$\checkmark$ Disseminação - é essa etapa que se preocupa em entregar a informação tratada e analisada para o usuário final, de modo que ele possa tomar as decisões mais adequadas.

Embora no Ciclo da IOC, os Sistemas de Informação baseados em Computadores sejam parte integrante do processo, é importante destacar que a etapa de planejamento, segundo Falsarella et al. (2014), é realizada por pessoas, pois somente os gestores no contexto do PEE poderão dizer quais serão os campos de interesse que a organização pretende observar, definir as demandas e as fontes de informação e descrever as necessidades e finalidades. Como exemplos de demandas e fontes de informação que poderiam ser trabalhados pelos gestores na etapa de Planejamento do Ciclo da IOC, observa-se o conteúdo adaptado a partir de autores como Porter (2004) e Oliveira (2014), conforme indicado no Quadro 2.

Quadro 2 - Fontes publicadas para análise do ambiente

\begin{tabular}{|c|c|c|}
\hline FONTE & INSTRUMENTO & INFORMAÇÕES \\
\hline ESPECIALISTAS E & - Livros/Banco de dados & - Estudos da indústria \\
\hline EMPRESAS & - Relatórios de pesquisa & - Dados estatísticos \\
\hline ESPECIALIZADAS & & $\begin{array}{l}\text { Análise e tendências da economia, } \\
\text { mercado e tecnologia }\end{array}$ \\
\hline ASSOCIAÇÕES COMERCIAIS & - Publicações próprias & $\begin{array}{l}\text { - Dados sobre infraestrutura, produção, } \\
\text { concorrentes etc. } \\
\text { - Estatísticas sobre a indústria }\end{array}$ \\
\hline
\end{tabular}




\begin{tabular}{|c|c|c|}
\hline $\begin{array}{l}\text { REVISTAS } \\
\text { ESPECIALIZADAS E } \\
\text { IMPRESSOS COMERCIAIS }\end{array}$ & $\begin{array}{ll}\text { - } & \text { Impressos } \\
\text { - } & \text { Publicações eletrônicas }\end{array}$ & $\begin{array}{l}\text { - Eventos, fornecedores, distribuidores, } \\
\text { companhias etc. }\end{array}$ \\
\hline GOVERNO & $\begin{array}{l}\text { - } \quad \text { Projetos Governamentais } \\
\text { - } \text { Registros de marcas e } \\
\text { patentes }\end{array}$ & $\begin{array}{l}\text { - } \text { Oportunidade de negócios } \\
\text { - Avanço tecnológico e os novos } \\
\text { produtos }\end{array}$ \\
\hline CONCORRENTES & 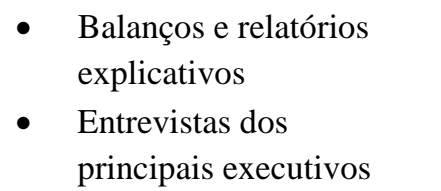 & $\begin{array}{l}\text { - Revelam a situação econômica - } \\
\text { financeira e principais projetos } \\
\text { - Revelam prioridades e propósitos da } \\
\text { empresa }\end{array}$ \\
\hline JORNAIS & $\begin{array}{l}\text { - Produtos e campanhas } \\
\text { mercadológicas }\end{array}$ & $\begin{array}{l}\text { Revelam planos de produção, novos } \\
\text { produtos e prioridades }\end{array}$ \\
\hline
\end{tabular}

Fonte: Adaptado de Porter (2004) e Oliveira (2014).

Assim, descobrir ameaças e oportunidades e forças e fraquezas, requer que os gestores responsáveis pela elaboração e acompanhamento do PEE, indiquem primeiramente que dados e informações devem ser observadas, sendo elas internas ou externas, estruturadas ou não estruturadas. Desse modo, como foi apresentado no Quadro 2, poderia ser relacionada a fonte, o instrumento e as informações com a finalidade de descrever onde e que dados e informações devem ser coletadas.

Voltando ao Ciclo da IOC, para Falsarella et al. (2014), um ponto importante que merece ser observado

[...] é que tanto a fase de Coleta de Dados e Informações, como a de Análise, pelo fato de serem realizadas com o apoio de sistemas de informação, devem ser automatizadas, permitindo, desse modo, avaliar um grande volume de dados e informações disponíveis no ambiente de atuação da organização (FALSARELLA et al., 2014).

A Figura 3, apresentada por Falsarella et al. (2014) e chamada de Sistema de Inteligência Organizacional e Competitiva (SIOC), representa o processo de coleta de dados e informações e análise realizados com o auxílio de Sistemas de Informação (SI), de modo a fornecer informações para auxiliar o processo decisório na elaboração e acompanhamento do PEE. Os autores descrevem o SIOC em cinco passos. São eles: 
Sistema de Inteligência Organizacional e Competitiva

Ciclo da IOC

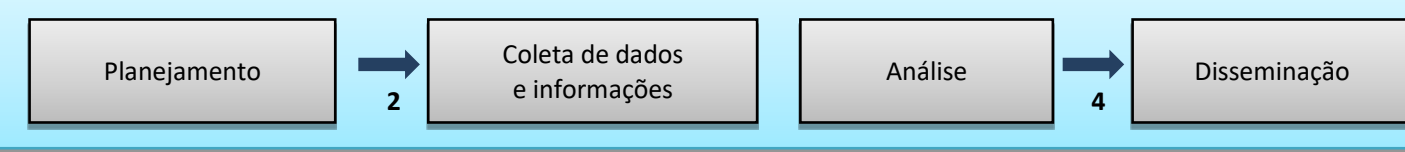

3

Sistemas de Informação

Transacionais, Gerenciais, de Apoio à Decisão, de Informação para Executivos e de Inteligência de Negócios

Estratégico Empresarial

Elaboração

Execução e

Acompanhamento
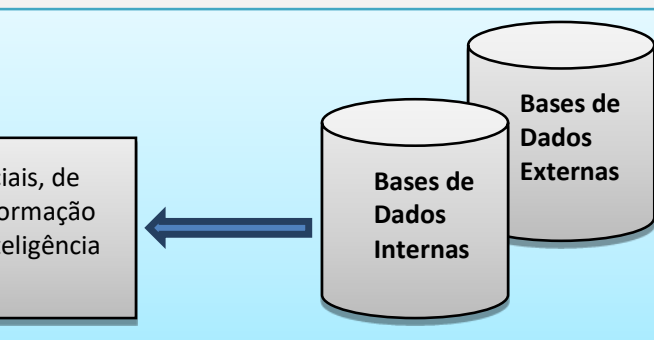

(n)

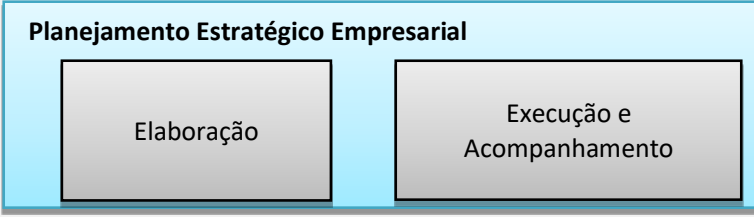

Figura 3 - Sistema de Inteligência Organizacional e Competitiva

Fonte: Falsarella et al. (2014).

1. Deve ser definido o campo de interesse que a organização pretende observar e as fontes de informação. Também deve ser identificado que dados e informações podem ser úteis levando a novas oportunidades e ameaças, além de sinalizar forças e fraquezas (Planejamento);

2. É realizada a coleta de dados e informações. Devem ser relacionados os Sistemas de Informação e as bases de dados internas e externas que serão úteis e que deverão fornecer os dados e informações;

3. Com os dados e informações, é possível iniciar a etapa de Análise do Ciclo da IOC onde, após a análise e busca de relações, podem emergir ameaças e oportunidades e forças e fraquezas;

4. Inicia-se a Disseminação, onde a informação tratada e analisada será entregue para os usuários;

5. Toma-se a decisão mais adequada contribuindo, assim, com o processo de elaboração do PEE. 
Paralelamente à execução do PEE, inicia-se a etapa de acompanhamento do plano, onde o SIOC pode fornecer informações e subsídios para que seja verificado pelos gestores se as estratégias adotadas estão dando resultado.

Analisando o SIOC percebe-se que ele pode ser ampliado e evoluído e ser transformado em um Modelo de Monitoramento Informacional, principalmente se a ele forem agregados os conceitos e características de Big Data e Agente de Software Inteligente (vide Figura 4).

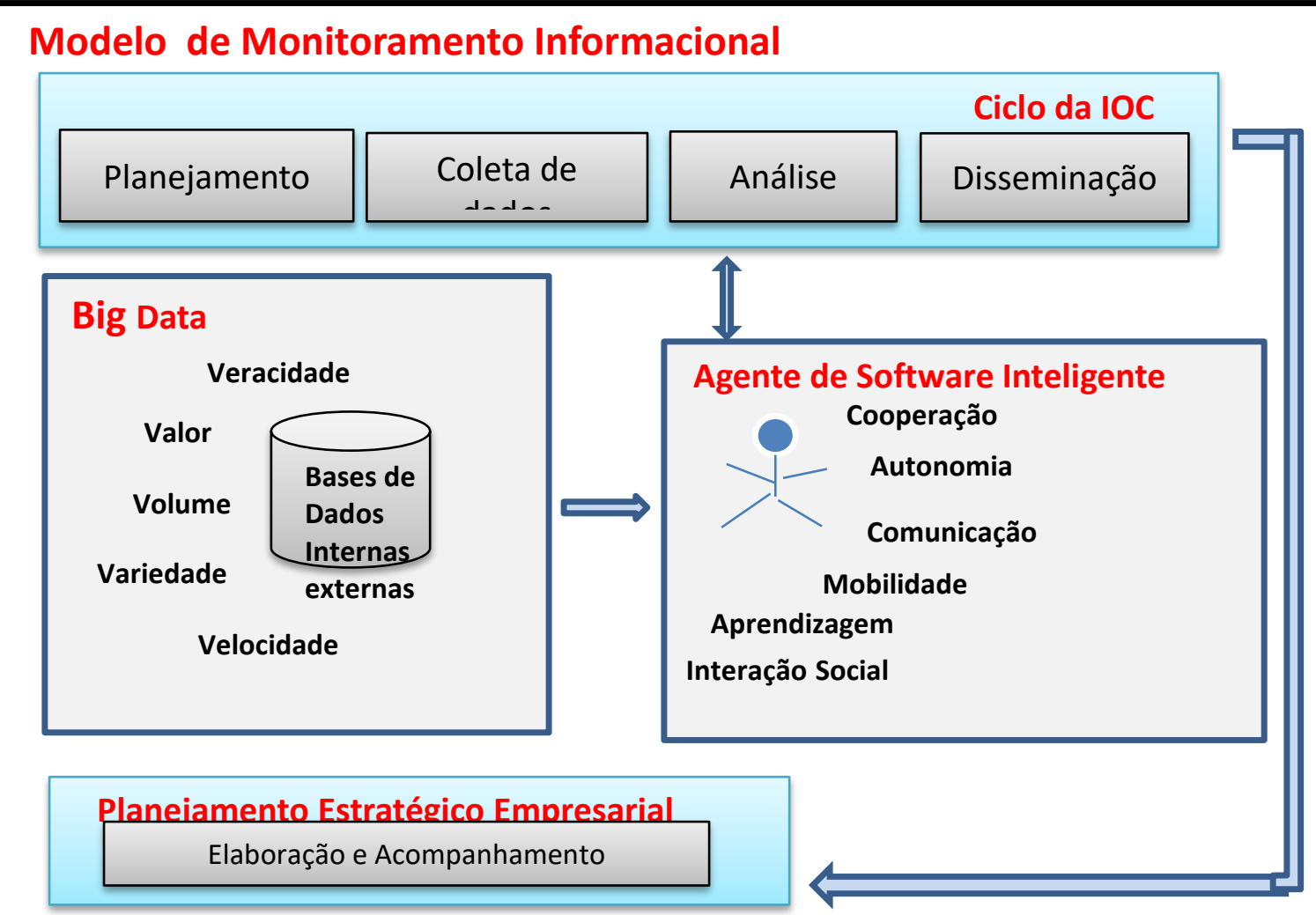

Figura 4 - Modelo de Monitoramento Informacional Fonte: Autores deste trabalho.

Para essa ampliação, evolução e transformação, os 5 "Vs" do Big Data, descritos na Figura 1, serão associados com as fases da IOC e com as características dos ASI, de modo que a relação apresentada no Quadro 3, possa contribuir para descrever a seguir o Modelo de Monitoramento Informacional proposto. 
Quadro 3 - Relação Big Data, IOC e Agentes de Software

\begin{tabular}{|cll|}
\hline Big Data 5 Vs & \multicolumn{1}{c|}{ Fases da IOC } & $\begin{array}{l}\text { Características dos Agentes de } \\
\text { Software Inteligentes }\end{array}$ \\
\hline Variedade & $\begin{array}{l}\text { Planejamento, Coleta de Dados } \\
\text { e Informações }\end{array}$ & $\begin{array}{l}\text { Autonomia, cooperação e } \\
\text { comunicação }\end{array}$ \\
\hline Volume & Coleta de Dados e Informações & $\begin{array}{l}\text { Autonomia, Cooperação, } \\
\text { Comunicação e Mobilidade }\end{array}$ \\
\hline Veracidade & Planejamento e Análise & $\begin{array}{l}\text { Aprendizagem, autonomia, } \\
\text { Cooperação, Comunicação }\end{array}$ \\
& Planejamento e Análise & $\begin{array}{l}\text { Aprendizagem, autonomia, } \\
\text { Interação Social }\end{array}$ \\
\hline Valor & Planejamento, Coleta de Dados & $\begin{array}{l}\text { Autonomia, Cooperação, } \\
\text { Comunicação e Mobilidade }\end{array}$ \\
\hline Velocidade & Informações & \\
\hline
\end{tabular}

Fonte: Autores deste trabalho.

Uma vez na etapa de Planejamento do Ciclo da IOC tendo sido estabelecido o campo de interesse que uma organização pretende observar, definida a demanda e as fontes de informação, o ASI pode iniciar a etapa de Coleta de Dados e Informações e simultaneamente ou posteriormente a de Análise.

Com relação à Variedade de informações, os locais onde o ASI (estático ou móvel) deve pesquisar e coletar são definidos na fase de Planejamento da IOC, sejam os dados ou informações, internos ou externos à empresa, estruturados ou não estruturados. Com esse requisito definido, o ASI terá autonomia para realizar a tarefa a ele delegada, inclusive podendo se comunicar e ter a cooperação de outros agentes.

Com relação à quantidade de informações existentes a ser explorada (Volume), por ser uma entidade de software com autonomia, que poderá se comunicar e ter a cooperação de outros agentes, inclusive possuir a característica de mobilidade para viajar por vários servidores da rede, essa tarefa será realizada por um ASI em muito menos tempo do que um Ser Humano. Assim, sem dúvida alguma, essa é uma grande vantagem do ASI.

Já a Veracidade, no sentido de extrair dados e informações confiáveis, descartando os não confiáveis, de uma variedade e volume muito grande de informações, é uma tarefa bastante difícil de ser realizada por um ASI. No entanto, essa complexidade pode ser reduzida, se pensarmos que as informações internas, normalmente estruturadas, são confiáveis. Além disso, na fase de Planejamento da IOC, os gestores do PEE podem definir locais de busca que sejam confiáveis.

\begin{tabular}{c|c|c|c|c|c}
\hline (C) RDBCI: Rev. Digit. Bibliotecon. Cienc. Inf. & Campinas, SP & v.15 & n.2 & p. 420-441 & maio/ago. 2017 \\
\hline
\end{tabular}


Somado a isso, considerando a característica de autonomia, a cooperação e comunicação com outros agentes podem trazer informações de locais de busca não confiáveis, contribuindo para a aprendizagem do ASI, ou seja, uma vez detectado por vários agentes que um local não é confiável, ele pode usar a sua característica de Aprendizagem para evitar coletar informações desse local.

Quanto ao Valor e a consequente geração de insights quando da análise das informações, esse é o processo mais complexo para a definição dos requisitos do ASI. No entanto, de posse dos dados e informações coletadas, pode-se pensar que esse processo é semelhante a um Sistema de Apoio à Decisão. Portanto, o ASI pode, conforme foi descrito no Quadro 1, ser personalizado para cada usuário e aplicação, possuir diferentes representações, dividindo o problema e os sistemas de raciocínio em partes menores, aprender com o usuário, tratar fatores e situações dinamicamente e possuir um comportamento de busca e escolha construtiva, reforçando as características de aprendizagem, autonomia e interação social.

Quanto a Velocidade com que os dados e as informações aumentam e se modificam, a fase de Planejamento da IOC deve fornecer novos locais de coleta que serão somados aos existentes, podendo o agente com as características de autonomia, cooperação, comunicação e mobilidade, descobrir rapidamente eventuais mudanças que estão ocorrendo no ambiente onde a empresa atua.

Finalmente, o ASI ao finalizar as etapas de Coleta de Dados e Informações e de Análise, as informações tratadas e analisadas podem ser entregues na fase de Disseminação para o usuário final, de modo que ele possa tomar as decisões mais adequadas quanto a gestão estratégica empresarial.

\section{CONSIDERAÇÕES FINAIS}

O objetivo deste trabalho foi propor um Modelo de Monitoramento Informacional para subsidiar a gestão estratégica empresarial, de modo a identificar ameaças e oportunidades e forças e fraquezas, informações importantes para a elaboração e acompanhamento do PEE. Diante disso, buscou-se a associação de quatro conceitos, ou seja, Gestão Estratégica, Inteligência Organizacional e Competitiva, Big Data e Agente de Software inteligente.

Ao descrever os conceitos, percebeu-se que era possível criar o Modelo a partir da ampliação e evolução de um Sistema de Inteligência Organizacional e Competitiva, onde, as características dos 5 Vs encontrados no Big Data e as características do ASI, se automatizadas, poderiam contribuir com o processo de busca e análise de dados e informações relevantes para a elaboração e acompanhamento do PEE. 
Assim, aspectos como volume, variedade, confiabilidade dos dados e informações, associados à necessidade de se agregar algum valor à gestão estratégica empresarial, ganham uma nova dimensão quando se incrementa os processos de coleta, recuperação e análise com um Agente de Software Inteligente. Pelo fato de poder ser construído com características que permitam ter autonomia, aprender, cooperar e se comunicar com outros agentes, além de ter mobilidade e interagir com pessoas, o ASI é mais do que uma simples automatização dos processos. É o uso da inteligência para subsidiar de forma determinante a gestão e tomada de decisão na organização.

É nesse contexto que o Modelo de Monitoramento Informacional proposto pode atender vários seguimentos empresariais e situações diversas. Ele pode ser aplicado, por exemplo, no monitoramento do ambiente externo da organização (concorrência, novos produtos etc.); no acompanhamento de mudanças e tendências do mercado; no monitoramento do faturamento; no acompanhamento de índice de satisfação dos consumidores; entre outros.

Finalmente, vale destacar que a estrutura do modelo proposto ressalta a importância da integração do conceito Big Data com a ferramenta Agente de Software Inteligente no contexto do Ciclo de Inteligência Organizacional e Competitiva, de forma a agregar valor ao processo de Planejamento Estratégico das organizações que almejam se tornar cada vez mais competitivas.

\section{REFERÊNCIAS}

ALVES, Robson de Paula; FALSARELLA, Orandi Mina. Modelo conceitual de inteligência organizacional aplicada à função manutenção. Gestão e produção, São Carlos, v. 16, n. 2, p. 313-324, abr./jun. 2009.

ANDERSON, Lisa; RÖNNBOM, Åsa. Intelligent agents: a new technology for future distributed sensor systems?. 2009. Master Thesis (Informatics Master) - Department of Informatics, School of Economies and Commercial Law, Göteborg University, Gothenburg, 2009.

AGARWAL, Puneet; SHROFF, Gautam; MALHOTRA, Pankaj. Approximate Incremental Big-Data Harmonization. In: IEEE INTERNATIONAL CONGRESS ON BIG DATA, 2., 2013, Santa Clara, CA. Anais eletrônicos... Santa Clara, CA, 2009. Disponível em: < http://ieeexplore.ieee.org/xpl/mostRecentIssue.jsp?punumber=6596172> Acesso em: $10 \mathrm{dez}$. 2014.

ARON, Ravi; SUNDARARAJAN, Arun; VISWANATHAN, Siva. Intelligent agents in electronic markets for information goods: customization, preference revelation and pricing. Decision Support Systems, Atlanta, v. 41, n. 4, p. 764-786, may, 2006.

\begin{tabular}{l|c|c|c|c|c}
\hline (C) RDBCI: Rev. Digit. Bibliotecon. Cienc. Inf. & Campinas, SP & v.15 & n.2 & p. 420-441 & maio/ago. 2017 \\
\hline
\end{tabular}


BARFIELD, Woodrow. Issues of law for software agents within virtual environments. MIT Press Journals, Cambridge, v. 14, n. 6, p. 741-748, mar. 2006.

BEGOLI, Edmon; HOREY, James. Design principles for effective knowledge discovery from big data. In: SOFTWARE ARCHITECTURE (WICSA) AND EUROPEAN CONFERENCE ON SOFTWARE ARCHITECTURE (ECSA), 2012, Helsink. Anais eletrônicos... IEEE/IFIP, 2012. p. 215-218.

BEULKE, Dave. Big data impacts data management: the 5 Vs of big data [blog post]. Dave Beulke Blog, 2011. Disponível em: 〈http:// davebeulke.com/big-data-impacts-datamanagement-the-five-vs-of-big-data> Acesso em: 15 jul. 2014.

BORKAR, Vinayak R.; CAREY, Michael J.; LI, Chen. Big data platforms: What's next?. XRDS: crossroads, The ACM magazine for students, v. 19, n. 1, p. 44-49. sept./nov.2012.

DEMIRKAN, Haluk; DELEN, Dursun. Leveraging the capabilities of service-oriented decision support systems: putting analytics and big data in cloud. Decision Support Systems, Atlanta, v. 55, n. 1, p. 412-421, 2013.

FALSARELLA, Orandi Mina; JANNUZZI, Celeste Aída Sirotheau Corrêa; SUGAHARA, Cibele Roberta. Planejamento estratégico empresarial: proposta de um sistema de inteligência organizacional e competitiva. RDBCI: Revista Digital de Biblioteconomia e Ciência da Informação, Campinas, v. 12, n.2, p. 193-216, 2014.

HAN, Xiaoyue et al. A Big Data model supporting information recommendation in social networks. In: INTERNATIONAL CONFERENCE ON CLOUD AND GREEN COMPUTING (CGC), 2., 2012. Anais eletrônicos... Hunan: IEEE, 2012. p. 810-813.

HICKMAN, Trey. Thanks for the memory: cooperative autonomous agent search in uncertain environments. Computers in Human Behavior, Atlanta, v. 26, n. 6, p. 1266-1275, nov. 2010.

JENNINGS, Nicholas R. On agent-based software engineering. Artificial Intelligence, v.117, n. 2, p. 277-296, mar.2000.

JI, Changqing et al. Big Data processing in cloud computing environments. In:

INTERNATIONAL SYMPOSIUM ON PERVASIVE SYSTEMS, ALGORITHMS AND NETWORKS (ISPAN), 12., 2012, San Marcos, Texas. Anais eletrônicos... San Marcos, Texas, IEEE, 2012. p. 17-23. Disponível em: < 
http://www.researchgate.net/publication/235933401_Big_Data_Processing_in_Cloud_Comp uting_Environments> Acesso em: 12 mar. 2014.

KAISLER, Stephen; ARMOUR, Frank; ESPINOSA, Alberto. Introduction to Big Data: Scalable Representation and Analytics for Data Science Minitrack. In: INTERNATIONAL CONFERENCE ON SYSTEM SCIENCES (HICSS), 46. 2013, Hawaii. Anais eletrônicos... Hawaii: IEEE, 2013. p. 984-984. Disponível em:< http://www.hicss.hawaii.edu/hicss_46/apahome46.htm> Acesso em: 23 jun. 2014.

KVASSOV, Vladimir. Strategic decisions and intelligent tools. In: HAWAII INTERNATIONAL CONFERENCE ON SYSTEM SCIENCES, 33. 2000, Hawaii. Anais eletrônicos... Hawaii: IEEE, 2000. p. 1-7. Disponível em:< http://ieeexplore.ieee.org/xpl/login.jsp?tp=\&arnumber=926666\&url=http\%3A\%2F\%2Fieeex plore.ieee.org\%2Fxpls\%2Fabs_all.jsp\%3Farnumber\%3D926666 > Acesso em: 23 jun. 2014.

LAKATOS, Eva Maria; MARCONI, Marina de Andrade. Metodologia do trabalho científico. 7 ed. São Paulo: Atlas, 2007.

MAES, Pattie apud DUAN, Yanqing et al. Supporting decision making process with "ideal" software agents-What do business executives want?. Expert Systems with Applications, Atlanta, v. 39, n. 5, p. 5534-5547, apr. 2012.

MITHAS, Sunil et al. Leveraging big data and business analytics [Guest editors' introduction]. IT professional, Los Alamitos/CA, v. 15, n. 6, p. 18-20, nov./dec. 2013. Disponível em < http://cloudcomputing.ieee.org/csdl/mags/it/2013/06/mit2013060018.pdf > Acesso em: mar. 2014.

MUNTEAN, Mihaela; TOMA, Cristian. Mobile agents systems in wireless applications. Journal of Mobile, Embedded and Distributed Systems, Bucareste, v. 1, n. 1, p. 13-19, 2009. Disponível em < http://www.jmeds.eu/index.php/jmeds/article/view/MobileAgents-Systems-in-Wireless-Applications> Acesso em: apr. 2014.

NÉRI, Edmilson Lucena. Agentes de software: delegando decisões a programas. RAEeletrônica, São Paulo, v. 4, n. 1, art. 3, jan./jul.2005.

OLIVEIRA, Djalma de Pinho Rebouças de. Sistemas de informações gerenciais: estratégicas, táticas, operacionais. 16. ed. São Paulo: Atlas, 2014.

OLIVEIRA, Letícia de. A estratégia organizacional na competitividade: um estudo teórico. REAd - Revista Eletrônica de Administração, Porto Alegre, v. 10., n. 4, jul./ago. 2004. 
PORTER, Michael Eugene. Estratégia competitiva: técnicas para análise de indústrias e da concorrência. 2. ed. Rio de Janeiro: Campus, 2004.

RESENDE, Solange Oliveira. Sistemas inteligentes: fundamentos e aplicações. Barueri: Manole, 2003.

SINGH, Sachchidanand; SINGH, Nirmala. Big Data Analytics. In: INTERNATIONAL CONFERENCE ON COMMUNICATION, 2012, Ottawa. Anais eletrônicos... Ottawa: Information \& Computing Technology (ICCICT), Oct. 19-20, 2012. p.1-4. Disponível em: < http://ieeexplore.ieee.org/xpls/abs_all.jsp?arnumber=6398180\&tag=1> Acesso em: jun. 2014.

SPROULE, Susan; ARCHER, Norman P. Knowledge agents for search and choice support in e-commerce: a decision support systems approach. Journal of Electronic Commerce Research, Taipei, v. 1, n.4, p. 152-165, 2000. Disponível em: < http://web.csulb.edu/journals/jecr/issues/20004/paper4.pdf> Acesso em: jun. 2014.

STAREC, Claudio; GOMES, Elisabeth; BEZERRA, Jorge (Org.). Gestão estratégica da informação e inteligência competitiva. São Paulo: Saraiva, 2005.

THOMPSON JR., Arthur A.; STRICKLAND III, A. J.; GAMBLE, John E. Administração estratégica. 15. ed. Porto Alegre: AMGH, 2008.

VALENTIM, M. L. P. et al. O processo de inteligência competitiva em organizações.

Datagramazero, Rio de Janeiro, v. 4, n. 3, p. 1-23, 2003.
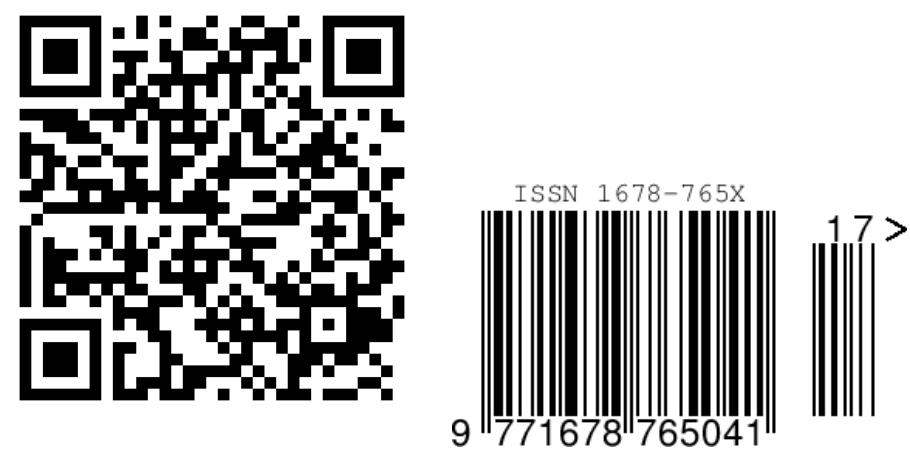